International Journal of Pure and Applied Mathematics

Volume 96 No. 1 2014, 15-25

ISSN: 1311-8080 (printed version); ISSN: 1314-3395 (on-line version)

url: http://www.ijpam.eu

doi: http://dx.doi.org/10.12732/ijpam.v96i1.2

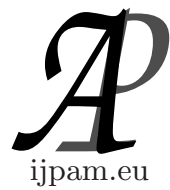

\title{
ON LINEAR AND MULTILINEAR MAPPINGS OF NUCLEAR TYPE
}

\author{
B. Martin Cerna Maguiña ${ }^{1 \S}$, Janet Mamani Ramos², \\ Héctor F. Cerna Maguiña ${ }^{3}$ \\ ${ }^{1}$ Department of Mathematics \\ National University of Santiago Antúnez de Mayolo \\ Campus Shancayan, Av. Centenario 200, Huaraz, PERÚ \\ ${ }^{2}$ Academic Department of Environmental and Natural Resources Engineering \\ National University of Callao \\ Av. Juan Pablo II 306, Callao, PERÚ \\ ${ }^{3}$ Departamento Académico de Contabilidad \\ Universidad Nacional Mayor de San Marcos \\ Av. Universitaria 306, Lima, Perú
}

\begin{abstract}
In this work, a technique is used in order to demonstrate the following lemmas "If $s \in \mathcal{F}(E, C(K))$, then $N_{\infty}^{0}(S)=\|S\|$ " and "If $S \in$ $\mathcal{F}\left(E, L_{p}(\Omega, \mu)\right)$, then $K_{p}^{0}(S)=\|S\|$ " and open question is answered in 18.1.16 which belong to Albert Pietsch, at the same time Lemma 2.1 of B. M. Cerna is demonstrated consequently additional results has been got.
\end{abstract}

Dedicated to the memory of our father: B. Cerna Figueroa

AMS Subject Classification: 47H60, 46G25, 47L20

Following the ideas of Pietsch, I couldn't extend the lemmas "If $s \in \mathcal{F}(E$, $C(K)$ ), then $N_{\infty}^{0}(S)=\|S\|$ " and "If $S \in \mathcal{F}\left(E, L_{p}(\Omega, \mu)\right)$, then $K_{p}^{0}(S)=\|S\|$ " toward the multilinear case. According to my interests fortunately, I could get

Received: February 20, 2014

(C) 2014 Academic Publications, Ltd.

$\S$ Correspondence author url: www.acadpubl.eu 
one technique based If $f \in X^{*}$ where $X$ is a normed space and $f \neq 0$, then $f$ is surjective and $X=\operatorname{ker} f \oplus M$, where $M$ is a one-dimensional subspace. These result and the theorem of Hahn Banach let me demonstrate the lemmas mentioned before, furthermore respond the question that is formulate in the book of Piestch in the lemma 18.1.16. furthermore this technique must have been extended to the multilinear case which let me get additional results.

A problem arise that It's more natural, There exist some relation between the theory that It served in the show of this results?

We introduce the notations in the present work, for Banach spaces $E_{1}, \cdots, E_{n}$ and $F$ over the field $\mathbb{K}(\mathbb{R}$ or $\mathbb{C})$, we denote $L\left(E_{1}, \cdots, E_{n} ; F\right)$ to the Banach space of all multi-linear and continuous applications of $E_{1} \times \cdots \times E_{n}$ over $F$ with a natural norm given by

$$
\|T\|=\sup _{\substack{x_{i} \in B_{E_{i}} \\ i=1, \cdots, n}}\left\|T\left(x_{1}, \cdots, x_{n}\right)\right\|
$$

where $B_{E_{i}}$ denote the unitary ball of $E_{i}$, centered in $0 . E_{k}^{*}$ denote the dual topological of $E_{k}, k=1, \cdots, n$.

For $s \in\langle 0,+\infty\rangle$ we denote by $l_{s}(F)$ (or $l_{s}, F=\mathbb{K}$ ), the vector space of all sequences $\left(y_{j}\right)_{j=1}^{\infty}$ of elements that belong to $F$ such that

$$
l_{s}\left(y_{j}\right)=\left\|\left(y_{j}\right)_{j=1}^{\infty}\right\|_{s}=\left[\sum_{j=1}^{\infty}\left\|y_{j}\right\|^{s}\right]^{1 / s}<+\infty .
$$

For $s \geq 1,\|\cdot\|_{s}$ is a norm, and for $s<1$, is a s-norm. In any case we have a complete metric vector space. We denote by $l_{s}^{w}(F)$ the vector space of all sequences $\left(y_{j}\right)_{j=1}^{\infty}$ of elements that belong to $F$ such that

$$
\left\|\left(y_{j}\right)_{j=1}^{\infty}\right\|_{w, s}=w_{s}\left(y_{j}\right)=\sup _{\varphi \in B_{F^{*}}}\left\|\left(\varphi\left(y_{j}\right)\right)_{j=1}^{\infty}\right\|_{s}<+\infty
$$

so, $\left(l_{s}^{w}(F),\|\cdot\|_{w, s}\right)$ is a metric vector space.

For $s=+\infty$, we consider $l_{\infty}(F)=l_{\infty}^{w}(F)$ as a Banach space of all sequences $\left(y_{j}\right)_{j=1}^{\infty}$ of elements of $F$ under the norm

$$
w_{\infty}\left(y_{j}\right)=\left\|\left(y_{j}\right)_{j=1}^{\infty}\right\|_{\infty}=\left\|\left(y_{j}\right)_{j=1}^{\infty}\right\|_{w, \infty}=\sup _{j \in \mathbb{N}}\left\|y_{j}\right\|
$$


Let $0<r \leq \infty, 1 \leq p, q \leq \infty$, and $1+\frac{1}{r} \geq \frac{1}{p}+\frac{1}{q}$. An operator $s \in L(E, F)$ is called $(r, p, q)$-nuclear if

$$
s=\sum_{i=1}^{\infty} \sigma_{i} a_{i} \otimes y_{i}
$$

con $\left(\sigma_{j}\right)_{j=1}^{\infty} \in l_{r},\left(a_{i}\right)_{i=1}^{\infty} \in w_{q^{\prime}}\left(E^{*}\right)$, and $\left(y_{i}\right)_{i=1}^{\infty} \in w_{p^{\prime}}(F)$. In the case $r=\infty$ let us suppose that $\left(\sigma_{i}\right) \in c_{0}$.

We put

$$
N_{(r, p, q)}(s):=\inf l_{r}\left(\sigma_{i}\right) w_{q^{\prime}}\left(a_{i}\right) w_{p^{\prime}}\left(y_{i}\right)
$$

where the infimun is taken over all so-called $(r, p, q)$-nuclear representations described above.

The class of all $(r, p, q)$-nuclear operators is denoted by $\mathcal{N}_{(r, p, q)}$. $\mathcal{F}(E, F):=$ Ideal of finite operators of finite range from $E$ onto $F$. For every operators $s \in \mathcal{F}(E, F)$ we put

$$
N_{(r, p, q)}^{0}(s)=N_{f,(r, p, q)}(s):=\inf l_{r}\left(\sigma_{i}\right) w_{q^{\prime}}\left(a_{i}\right) w_{p^{\prime}}\left(y_{i}\right)
$$

where the infimum is taken over all finite representations

$$
s=\sum_{i=1}^{n} \sigma_{i} a_{i} \otimes y_{i}
$$

for $r \in] 0,+\infty], p, q_{j} \in[1, \infty], k=1, \cdots, n$, such that $n+\frac{1}{r} \geq \frac{1}{p}+\frac{1}{q_{1}}+\cdots+\frac{1}{q_{n}}$, $T \in L\left(E_{1}, \cdots, E_{n} ; F\right)$ is called $\left(r, p ; q_{1}, \cdots, q_{n}\right)$-nuclear type and it takes the form

$$
T=\sum_{k=1}^{\infty} \sigma_{k} x_{k, 1}^{*} \times \cdots \times x_{k, n}^{*} \otimes y_{k}
$$

with $\left(\sigma_{j}\right)_{j=1}^{\infty} \in l_{r},\left(y_{k}\right)_{k=1}^{\infty} \in l_{p^{\prime}}^{w}(F)$ and $\left(x_{k, j}^{*}\right)_{k=1}^{\infty} \in l_{q_{j}^{\prime}}^{w}\left(E_{j}^{*}\right), j=1, \cdots, n$.

In the case $r=+\infty$ the condition for $\left(\sigma_{k}\right)_{k=1}^{\infty}$ is to be in $c_{0}$.

The set of such applications satisfying such definition is a vector space and is denote by $\mathcal{N}_{r, p, q_{1}, \cdots, q_{n}}\left(E_{1}, \cdots, E_{n} ; F\right)$. 
Considering that

$$
N_{\left(r, p, q_{1}, \cdots, q_{n}\right)}(T)=\inf l_{r}\left(\sigma_{k}\right) \prod_{j=1}^{n} w_{q_{j}^{\prime}}\left(x_{k, j}^{*}\right) w_{p^{\prime}}\left(y_{k}\right)
$$

where the infimum is taken over all possible representations of $T$ described in (3).

$$
L_{f}\left(E_{1}, \cdots, E_{n} ; F\right):=\text { multi-linear applications of finite type }
$$

For every operator $\psi \in L_{f}\left(E_{1}, \cdots, E_{n} ; F\right)$ we put

$$
N_{f,\left(r, p, q_{1}, \cdots, q_{n}\right)}(\psi)=\inf \left\|\left(\sigma_{k}\right)_{k=1}^{m}\right\|_{r} \prod_{j=1}^{n}\left\|\left(x_{k, j}^{*}\right)_{k=1}^{m}\right\|_{w, q_{j}^{\prime}}\left\|\left(y_{k}\right)_{k=1}^{m}\right\|_{w, p^{\prime}}
$$

where the infimum is taken over all finite representations

$$
\psi=\sum_{k=1}^{m} \sigma_{k} x_{k, 1}^{*} \times \cdots \times x_{k, n}^{*} \otimes y_{k}
$$

where $p^{\prime}$ is a dual exponent, $\frac{1}{p}+\frac{1}{p^{\prime}}=1 . K_{F}$ is the isometric immersion of $F$ into $F^{* *}$.

\section{Linear Operators of $(r, p, q)$-Nuclear Type}

In this section using the proposition 1.1 and one consequence of the HanhnBanach Theorem see [2] demonstrate the lemmas 19.2.5, 19.3.6 and to get similar result of 18.1.16. See [6].

Proposition 1.1. Lets $X$ a normed linear space over the field $\mathbb{K}$ ( $\mathbb{R}$ or $\mathbb{C}$ ), $f_{1}, f_{2} \in X^{*}$ with $f_{1}, f_{2}$ is linearly independent. Then exist $x \in X$ and $\lambda \in \mathbb{K}$ such that $f_{1}(x)=a$ and $f_{2}(x)=\lambda a$ or $f_{1}(x)=a$ and $f_{2}(x)=0$ where $a, \lambda \in \mathbb{K}$ and $a \neq 0$

Proof. Since $f_{1}, f_{2}$ is surjective there exist $x_{1}, x_{2} \in X$ such that $f_{1}\left(x_{1}\right)=a$, $f_{2}\left(x_{2}\right)=\beta a$. We know that $X=\operatorname{ker} f_{1} \oplus M_{1}=\operatorname{ker} f_{2} \oplus M_{2}$, where $M_{1}$ and $M_{2}$ are one dimensional subspaces $M_{1}=\left\langle m_{1}\right\rangle, M_{2}=\left\langle m_{2}\right\rangle$. Así

$$
\begin{aligned}
& x_{1}=y_{1}+\lambda_{1} m_{2} \quad, \quad y_{1} \in \operatorname{ker} f_{2}, m_{2} \in M_{2} \\
& x_{2}=y_{2}+\lambda_{2} m_{2} \quad, \quad y_{2} \in \operatorname{ker} f_{2}, m_{2} \in M_{2}
\end{aligned}
$$


Then

$$
\begin{gathered}
f_{2}\left(x_{1}\right)=\lambda_{1} f_{2}\left(m_{2}\right) \\
\beta a=f_{2}\left(x_{2}\right)=\lambda_{2} f_{2}\left(m_{2}\right)
\end{gathered}
$$

of (4) and (5) we have $f_{2}\left(x_{1}\right)=\beta \frac{\lambda_{1}}{\lambda_{2}} a$, to take $\lambda=\beta \frac{\lambda_{1}}{\lambda_{2}}$ for can get $f_{2}\left(x_{1}\right)=\lambda a$.

Lemma 1.1. Let $\psi: E \longrightarrow L_{p}(\Omega, \mu)$ be defined by

$$
\psi(x)=\sum_{k=1}^{m} \sigma_{k} x_{k}^{*}(x) y_{k}
$$

where $\frac{1}{p}+\frac{1}{q}=1,1 \leq p<\infty$, then

$$
N_{f,(\infty, p, q)}(\psi)=N_{(\infty, p, q)}(\psi)=\|\psi\|
$$

Proof. It is clear that for $\frac{1}{p}+\frac{1}{q}=1$, one has from (1) and (2)

$$
\|\psi\| \leq N_{(\infty, p, q)}(\psi) \leq N_{f,(\infty, p, q)}(\psi)
$$

Moreover

$$
\|\psi\|\|x\| \geq\left[\int_{\Omega}\left|\sum_{k=1}^{m} \sigma_{k} x_{k}^{*}(x) y_{k}(t)\right|^{p} d u(t)\right]^{\frac{1}{p}}
$$

Let

$$
M=\sup _{\|x\|=1}\left(\sum_{k=1}^{m}\left|\left\langle x_{k}^{*}, x\right\rangle\right|^{p}\right)^{\frac{1}{p}}
$$

it is clear that $M<+\infty$. From the proposition (1.1) there exist $\bar{x} \in E$ such that

$$
x_{k}^{*}(\bar{x})=\frac{M}{2^{\frac{k}{p}}}, \quad k=1, \cdots, m
$$

Therefore, from the relation (9) we have that, one has $\epsilon>0$, $M\|x\|<(1+\epsilon)\left(\sum_{k=1}^{m} \frac{M^{p}}{2^{k}}\right)^{\frac{1}{p}}$, which implies that $\|\bar{x}\|<1+\epsilon, \forall \epsilon>0$.

Therefore $\|\bar{x}\| \leq 1$, from the relation (8) one has

$$
\|\psi\| \geq\left[\int_{\Omega}\left|\sum_{k=1}^{m} \sigma_{k} \frac{M}{2^{\frac{k}{p}}} y_{k}(t)\right|^{p} d u(t)\right]^{\frac{1}{p}}
$$




$$
\|\psi\| \geq M\left[\int_{\Omega}\left|\sum_{k=1}^{m} \sigma_{k} \frac{1}{2^{\frac{k}{p}}} y_{k}(t)\right| d u(t)\right]^{\frac{1}{p}}
$$

Let

$$
z(t)=\sum_{k=1}^{m} \sigma_{k} \frac{y_{k}(t)}{2^{\frac{k}{p}}}
$$

them for $1 \leq p<\infty$ we have

$$
|\langle\phi, z\rangle|=\left|\sum_{k=1}^{m} \sigma_{k}\left\langle\phi, \frac{y_{k}}{2^{\frac{k}{p}}}\right\rangle\right| \leq\|\phi\|\|z\|
$$

In addition, let

$$
L=\operatorname{span}_{k \in\{1, \cdots, m\}-\left\{k_{0}\right\}}\left\{\frac{y_{k}}{2^{\frac{k}{p}}}\right\}
$$

Moreover, as consequence of the Hahn-Banach theorem (see [2]) there exists $\phi$ such that

$$
\|\phi\|=\frac{1}{d}, \quad\langle\phi, x\rangle=0, \text { for all } x \in L \text { and }\left\langle\phi, \frac{y_{k_{0}}}{2^{\frac{k_{0}}{p}}}\right\rangle=1
$$

where

$$
d=\inf _{x \in L}\left\|x-\frac{y_{k_{0}}}{2^{\frac{k_{0}}{p}}}\right\|
$$

and further can choose $\sigma_{k_{0}}$ such that

$$
\left|\sigma_{k_{0}}\right|=\max _{k=\overline{1, n}}\left|\sigma_{k}\right|=l_{\infty}\left(\sigma_{k}\right)
$$

Taking into account these last relations in the equation (11) we can get

$$
\|z\| \geq\left|\sigma_{k_{0}}\right| d
$$

Since

$$
x=\sum_{k=1, k \neq k_{0}}^{m}-\frac{y_{k}}{2^{\frac{k}{p}}} \in L
$$

then for a given $\epsilon>0$ one has

$$
(1+\epsilon) d>\left\|\sum_{k=1}^{m} \frac{y_{k}}{2^{\frac{k}{p}}}\right\|
$$


therefore, from the relations (12) one has:

$$
(1+\epsilon)\|z\|>l_{\infty}\left(\sigma_{k}\right)\left\|\sum_{k=1}^{m} \frac{y_{k}}{2^{\frac{k}{p}}}\right\|
$$

We know that

$$
w_{p^{\prime}}\left(y_{k}\right)=\sup _{a \in B_{l_{p}}^{m}}\left\|\sum_{k \leq m} a_{k} y_{k}\right\|
$$

and since

$$
a=\left(\frac{1}{2^{\frac{k}{p}}}\right)_{k=1}^{m} \in B_{l_{p}}^{m}
$$

for $\widetilde{\epsilon}>0$ we have

$$
(1+\widetilde{\epsilon})\left\|\sum_{k \leq m} \frac{1}{2^{\frac{k}{p}}} y_{k}\right\|>w_{p^{\prime}\left(y_{k}\right)}
$$

From the last relation an the equation (13) one obtains

$$
\|z\|(1+\epsilon)(1+\widetilde{\epsilon})>l_{\infty}\left(\sigma_{k}\right) w_{p^{\prime}}\left(y_{k}\right), \text { for all } \epsilon>0, \text { and } \widetilde{\epsilon}>0
$$

Therefore, from the relations (10) y (14) one has

$$
\|\psi\| \geq l_{\infty}\left(\sigma_{k}\right) w_{p^{\prime}}\left(y_{k}\right) M=N_{f,(\infty, p, q)}
$$

From equations (7) and (15) one has required result.

For $p=+\infty$, we have

$$
\|\psi\|=\sup _{\|x\|_{E}=1}\|\psi(x)\|_{L_{\infty}(\Omega, \mu)}
$$

where

$$
\|\psi(x)\|_{L_{\infty}(\Omega, \mu)}=\inf _{A>0}\{A /|\psi(x(t))| \leq A, \text { except for a set of measure zero }\}
$$

also we have:

$$
\|\psi\|\|x\|_{E} \geq\|\psi(x)\|_{L_{\infty}(\Omega, \mu)},
$$

for $\epsilon>0$, there exists $A>0$ and $N \subset \Omega$ with $\mu(N)=0$ such that

$$
\begin{gathered}
|\psi(x(t))|(1-\epsilon) \leq(1-\epsilon) A<\|\psi(x)\|_{L_{\infty}(\Omega, \mu)} \\
\|\psi\|\|x\|_{E}>(1-\epsilon)|\psi(x(t))|, \forall t \in(\Omega-N)
\end{gathered}
$$


From the equations (4) and (16) one can get

$$
\|\psi\|\|x\|_{E}>(1-\epsilon)\left|\sum_{k=1}^{m} \sigma_{k} x_{k}^{*}(x) y_{k}(t)\right|, \quad \forall t \in(\Omega-N)
$$

From the proposition (1.1) there exists $\bar{x} \in E$ such that

$$
\|\bar{x}\|_{E} \leq 1 \text { y } x_{k}^{*}(\bar{x})=\frac{M}{2^{\frac{k}{p}}}=M, \text { with } p=\infty
$$

where

$$
M=\sup _{\|x\|_{E}=1} \sup _{k \in\{1, \cdots, m\}}\left|\left\langle x_{k}^{*}, x\right\rangle\right|
$$

Then it is clear that $M<+\infty$ and of (17) we have

$$
\|\psi\|>(1-\epsilon) M\left|\sum_{k=1}^{m} \sigma_{k} y_{k}(t)\right|, \forall t \in(\Omega-N)
$$

Let

$$
z(t)=\sum_{k=1}^{m} \sigma_{k} y_{k}(t), \quad \forall t \in(\Omega-N)
$$

we have

$$
|\langle\varphi, z\rangle|=\left|\sum_{k=1}^{m} \sigma_{k}\left\langle\varphi, y_{k}\right\rangle\right| \leq\|\varphi\|\|z\|
$$

In addition, let

$$
L=\operatorname{span}_{k \in\{1, \cdots, m\}-\left\{k_{0}\right\}}\left\{y_{k}\right\}
$$

Moreover, as a consequence of the Hahn-Banach theorem (see [2]), there exists $\varphi$ such that

$$
\|\varphi\|=\frac{1}{d},\langle\varphi, x\rangle=0, \text { for all } x \in L \text { and }\left\langle\varphi, y_{k_{0}}\right\rangle=1
$$

where

$$
d=\inf _{x \in L}\left\|x-y_{k_{0}}\right\|
$$

and further one can choose $\sigma_{k_{0}}$ such that

$$
\left|\sigma_{k_{0}}\right|=\max _{k=1, m} \sigma_{k}=l_{\infty}\left(\sigma_{k}\right)
$$


Taking into account these last relations in equation (19) one can get

$$
\|z\| \geq\left|\sigma_{k_{0}}\right| d
$$

since

$$
x=\sum_{\substack{k=1 \\ k \neq k_{0}}}^{m}-y_{k} \text { and } x \in L
$$

then

$$
(1+\epsilon) d>\left\|\sum_{k=1}^{m} y_{k}\right\|
$$

Therefore, from the relations (20) one has:

$$
(1+\epsilon)\|z\|>l_{\infty}\left(\sigma_{k}\right)\left\|\sum_{k=1}^{m} y_{k}\right\|
$$

we know that

$$
w_{1}\left(y_{k}\right)=\sup _{a \in B_{l_{\infty}}^{m}}\left\|\sum_{k \leq m} a_{k} y_{k}\right\|
$$

since

$$
a=\{1\}_{k=1}^{m} \quad \mathrm{y} \quad a \in B_{l_{\infty}}^{m}
$$

For $\widetilde{\epsilon}>0$ we have that

$$
(1+\widetilde{\epsilon})\left\|\sum_{k \leq m} y_{k}\right\|>w_{1}\left(y_{k}\right)
$$

From the relations (21) and (22) we have:

$$
(1+\epsilon)(1+\widetilde{\epsilon})\|z\|>l_{\infty}\left(\sigma_{k}\right) w_{1}\left(y_{k}\right), \text { for all } \epsilon>0 \text {, and } \widetilde{\epsilon}>0
$$

Therefore, from the last relations and (18) we have

$$
\|\psi\| \geq M l_{\infty}\left(\sigma_{k}\right) w_{1}\left(y_{k}\right)=N_{f,(\infty, \infty, 1)}(\psi)
$$

From equations (5) and (23) one has the required result.

$$
N_{f,(\infty, \infty, 1)}(\psi)=N_{(\infty, \infty, 1)}(\psi)=\|\psi\|
$$


Therefore, for $p=\infty$, one has the following

Lemma 1.2. Let $K$ be any compact Hausdorff space. Then for $S \in$ $\mathcal{F}(E, C(K))$, we have $N_{(\infty, \infty, 1)}(\psi)=N_{f,(\infty, \infty, 1)}(\psi)=\|\psi\|$.

In the Subsection 18.1.16 Pietsch say for Special exponents the above result holds without any assumption on the underlying Banach space.

Proposition 1.2. $[6] N_{(r, 2, q)}^{0}(s)=N_{(r, 2, q)}(s)$ for all $s \in \mathcal{F}(E, F)$.

Proof. (See [6])

Proposition 1.3. $N_{f,(\infty, p, q)}(s)=N_{(\infty, p, q)}(s)$ for all $s \in \mathcal{F}(E, F)$.

Proof. See lemma (1.1) on this article.

\section{Multilinear Mappings of Nuclear Type}

The next results is slightly different from the one given in lemma (1.1) and its proof can be performed following the lines of this reference.

Lemma 2.1. [4] Let $\psi: E_{1} \times \cdots \times E_{n} \longrightarrow L_{p}(\Omega, \mu)$ defined by

$$
\psi\left(x_{1}, \cdots, x_{n}\right)=\sum_{k=1}^{m} \sigma_{k} x_{1, k}^{*}\left(x_{1}\right) \cdots x_{n, k}^{*}\left(x_{n}\right) y_{k}
$$

where $\frac{1}{p}=\frac{1}{q_{1}^{\prime}}+\cdots+\frac{1}{q_{n}^{\prime}}$.

Then $N_{f,\left(\infty, p, q_{1}, \cdots, q_{n}\right)}(\psi)=N_{\left(\infty, p, q_{1}, q_{n}\right)}(\psi)=\|\psi\|$.

Proof. (See [4])

Lemma 2.2. Let $\psi: E_{1} \times \cdots \times E_{n} \longrightarrow F$. If $\psi \in L_{f}\left(E_{1}, \cdots, E_{n} ; F\right)$, then

$$
N_{f,\left(\infty, p, q_{1}, \cdots, q_{n}\right)}(\psi)=N_{\left(\infty, p, q_{1}, \cdots, q_{n}\right)}(\psi)=\|\psi\|
$$

Lemma 2.3. Let $\psi: E_{1} \times \cdots \times E_{n} \longrightarrow F$. If $\psi \in L_{f}\left(E_{1}, \cdots, E_{n} ; F\right)$, then

$$
N_{\left(\infty, p ; q_{1}, \cdots, q_{n}\right)}(\psi)=N_{f,\left(\infty, p ; q_{1}, \cdots, q_{n}\right)}\left(K_{F} \psi\right)
$$




\section{Acknowledgments}

The author thanks M. C. Matos for the suggestions and Miguel Yglesias J. for reading the manuscript.

\section{References}

[1] R. Alencar, M. C. Matos, Some classes of multilinear mappings between Banach Spaces, publ. Dep. Análisis Matemático, Universidad Complutense de Madrid, Section 1, Number 12 (1989).

[2] S. Banach, Theory of Linear Operations, North Holland (1987).

[3] B. M. Cerna, Operadores Multilineares p-Fatorables, Ph.D. Thesis (2005), IMECC-UNICAMP, Campinas, Brazil; http://libdigi.Unicamp$\mathrm{br} /$ document $/$ ?code $=\mathrm{vt} 15000365809$

[4] B. M. Cerna, Some properties of Multi-Linear Operators of Nuclear Type, IJPAM. Volumen 56 N $N_{0} .1,2009-143-154$.

[5] M. C. Matos, On Multilinear Mappings of Nuclear Type, mathematic. Magazine of University Complutense of Madrid, 6, $N_{0} .1$ (1993)

[6] A. Pietsch, Operator Ideals, North-Holland Publishing Company, Amsterdan (1980) 
